Case Report

\section{Intestinal malrotation presenting with midgut volvulus in an adult}

\section{Marta Alberich Prats*, Ricard Frago Montanuy and Sebastiano Biondo Aglio}

Department of General and Gastrointestinal Surgery, Bellvitge University Hospital, Hospitalet de Llobregat, Spain

\section{Case Report}

A 36-year-old man without previous medical history presented to the emergency department with abdominal pain and vomiting of acute onset. His vital signs were stable and he had no fever. Abdominal exploration revealed distention with pain at palpation. Laboratory tests were normal. Abdominal $\mathrm{X}$-Ray showed gastric and small intestine distention. A nasogastric tube was placed and fluid therapy started. Computed tomography showed an intestinal malrotation, without the duodenum crossing the midline and with the colic frame arranged in the left hemiabdomen (Figure 1), complicated with a midgut volvulus as demonstrated by the whirlpool sign (Figure 2, arrow). Ladd's procedure was performed using a midline open approach. Mesenteric lymphadenopathy and chylous ascites was found (Figure 3). The volvulus was reduced by counter-clockwise detorsion without need of intestinal resection and Ladd's bands division along with an extended Kocher manoeuvre (Figure 4) allowed a good position of the mesentery. Appendectomy was also conducted. Postoperative course was uneventful with the

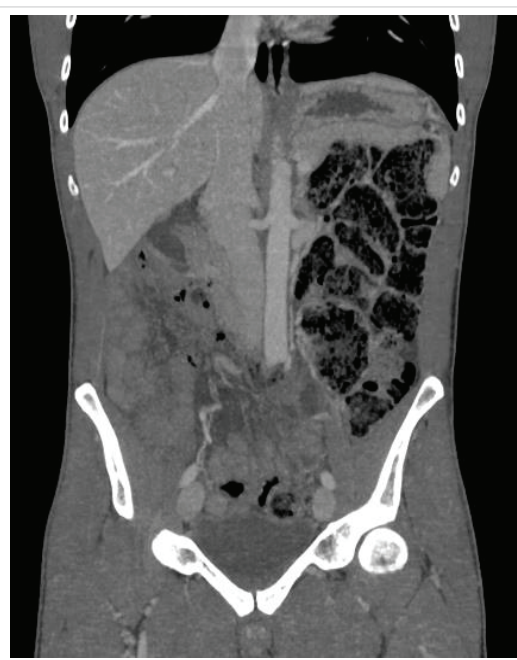

Figure 1: Abdominal computed tomography. Coronal section. Abnormal disposition of the small intestine, all of it located in the right hemiabdomen, and of the colon, rejected to the left hemiabdomen.

\section{More Information}

*Address for Correspondence: Marta Alberich Prats, Department of General and Gastrointestinal Surgery, Bellvitge University Hospital, Hospitalet de Llobregat, Spain, Tel: +34607245902;

Email: malberich94@hotmail.com

Submitted: 19 September 2019

Approved: 15 October 2019

Published: 16 October 2019

How to cite this article: Prats MA, Montanuy RF, Aglio SB. Intestinal malrotation presenting with midgut volvulus in an adult. Arch Case Rep. 2019; 3: 038-039.

DOI: dx.doi.org/10.29328/journal.acr.1001020

Copyright: () 2019 Prats MA, et al. This is an open access article distributed under the Creative Commons Attribution License, which permits unrestricted use, distribution, and reproduction in any medium, provided the original work is properly cited.

\section{(W) Check for updates}

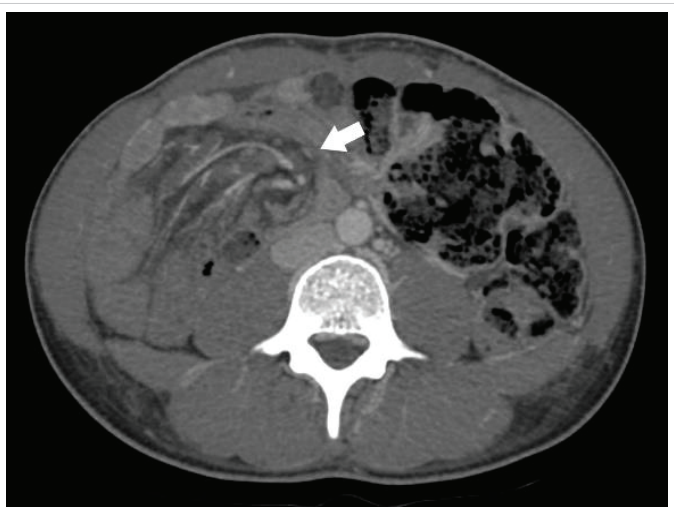

Figure 2: Abdominal computed tomography. Axial section. Whirlpool sign (arrow) due to rotation of the bowel around its mesentery leading to whirls of the mesenteric vessels.

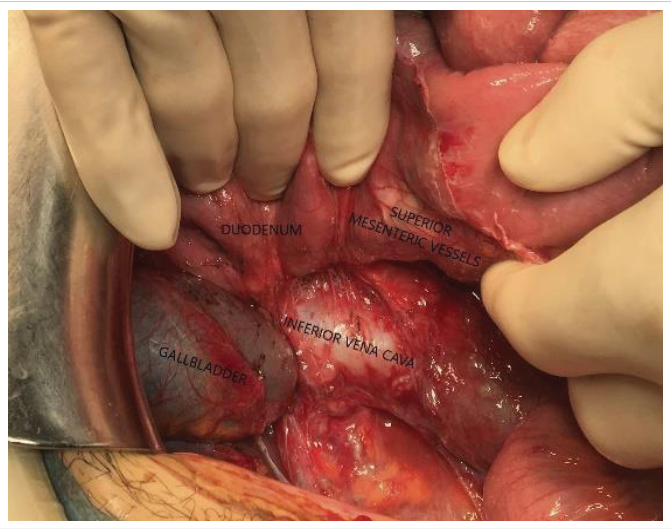

Figure 3: Intraoperative finding of mesenteric lymphadenopathy as a consequence of intestinal lymphatic drainage obstruction. 


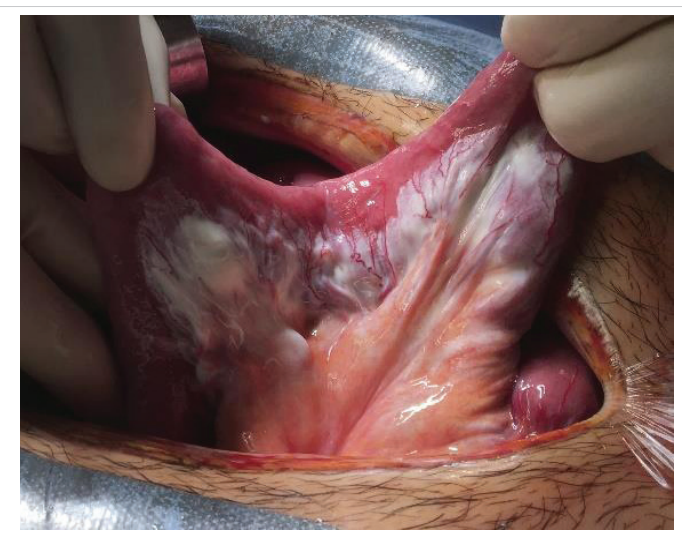

Figure 4: Intraoperative image showing an extensive kocher manoeuvre, which was necessary to restore a flat position of the mesentery.

patient being discharged the fifth day, after resumption of bowel function. No alterations were detected at follow up one month later.

\section{Discussion}

Intestinal malrotation is a rare fetal developmental abnormality which may go unnoticed until adulthood. It can be an incidental finding or present with mild symptoms, but it can also lead to an intestinal obstruction or acute ischemia due to a midgut torsion requiring emergency surgery [1]. Ladd's procedure is the standard treatment. It was described in 1936 and consists in a repositioning of the intestine by lysing the abnormal fixations. Since it is an uncommon situation and due to challenging anatomy and possible difficulties in reducing the volvulus an open approach is usually preferred. Appendectomy is recommended in order to avoid failure or delay in diagnosis $[2,3]$.

\section{References}

1. Kafadar MT, Cengiz AY, Çavis T, Bilgiç İ, Nadir I. Incidental intestinal malrotation in an adult: Midgut volvulus. Turk J Surg. 2018; 34: 337339. PubMed: https://www.ncbi.nlm.nih.gov/pubmed/30664437

2. Ladd W. Surgical Diseases of the Alimentary Tract in Infants. New England Journal of Medicine. 1936; 215: 705-708.

3. Tan $\mathrm{V}$, Kotobi $\mathrm{H}$, Parc $\mathrm{Y}$. Ladd procedure for malrotation with total intestinal volvulus. J Visc Surg. 2017; 154: 197-201. PubMed: https:// www.ncbi.nlm.nih.gov/pubmed/28522281 